\title{
Quality-of-Life Assessment in Patients Receiving Palliative Chemotherapy: Call for Action
}

\author{
Maheswari Senthil, MD, FACS $(1)$ \\ Division of Surgical Oncology, University of California Irvine, Orange, CA
}

Management of advanced, unresectable gastrointestinal cancers with palliative chemotherapy (PC) is a common and widely accepted oncologic practice as it is associated with modest survival benefit. ${ }^{1-3}$ In addition to survival gain, the key objectives of PC should also be to improve symptoms and preserve quality of life (QOL). In a shared decision-making model, data regarding the impact of $\mathrm{PC}$ on symptom improvement and maintenance of QOL will greatly influence patients' decision regarding PC. Furthermore, knowledge about disease-specific evolution of symptoms during the course of PC will improve the ability to anticipate problems, avert emergency room visits, and avoid deterioration of QOL.

In the article by Merchant et al., ${ }^{4}$ the authors have elegantly leveraged the advantage of a universal health care system and implementation of the Edmonton Symptom Assessment System (ESAS) as part of the Provincial Palliative Care Integration Project, to study symptom evolution in patients with gastric/esophageal cancer during PC. The ESAS covers nine domains (anxiety, depression, drowsiness, tiredness, lack of well-being, nausea, lack of appetite, pain, and shortness of breath), with scores for each domain reported separately rather than as a cumulative score, which renders the tool uniquely advantageous to assess the evolution of individual symptoms. In a cohort of 1900 patients, each with one or more recorded ESAS scores, the authors were able to discover some very important findings.

(C) Society of Surgical Oncology 2020

First Received: 13 October 2020

Accepted: 16 October 2020;

Published Online: 2 November 2020

M. Senthil, MD, FACS

e-mail: m.senthil@uci.edu
The highest symptom burden was observed in areas of appetite, tiredness, and well-being, all of which improved after PC. This important observation provides evidence regarding the role of $\mathrm{PC}$ in the improvement of symptoms and well-being, even in the absence of robust survival benefit in advanced gastric/esophageal cancers. Similar findings of symptom improvement were reported in a Korean study of PC in patients with advanced metastatic gastric cancer. ${ }^{5}$ Despite improvement in other domains, there was progressive deterioration of anxiety and depression scores throughout the course of PC. Psychosocial support to cope with the inevitable brevity of life in this group of patients is of paramount importance and should begin from the initial encounter, as deterioration in psychological domain negatively influences functioning in all other domains. ${ }^{6}$ Ethnic and cultural differences strongly influence the expression of anxiety and depression and should be taken into account to provide culturally sensitive psychosocial support. ${ }^{7,8}$ In the study by Merchant et al., ethnic distribution/differences were not highlighted but could be useful information in the design of strategies to address the psychological domain in patients receiving PC.

Another intriguing finding is that females were more likely to report moderate to severe scores of anxiety and depression (odds ratio 1.58, 95\% confidence interval 1.21-2.08) compared with males. We need to examine this difference at a deeper level. Our society systematically discourages and ridicules expression of emotional pain, fear, and anxiety by men, beginning from their early childhood. Phrases like, 'don't cry, be a man' and 'man up' have resulted in the indoctrination of a false notion that 'real men have no fear'. Hence, the negative judgment perceived or feared by men if and when they express emotional vulnerability might be an important reason as to why the reporting of anxiety/depression is lower in men. ${ }^{9,10}$ It is important to evaluate surrogate manifestations 
of anxiety and depression in men, as the lower rates of anxiety/depression could just be due to lower reporting rather than actual incidence.

Although the study by Merchant et al. used opportunistic ESAS scores rather than specific timepoints-based QOL assessments, it has clearly highlighted the importance of understanding symptom evolution during PC in patients with advanced cancers to implement appropriate strategies to maintain QOL. This study also provides an opportunity to unveil the regrettably poor reporting of QOL endpoints in major clinical trials in advanced cancers. For example, the TOGA, REGATTA, and AIO-FLOT 3 trials, important phase II/III studies in the treatment of patients with advanced/metastatic gastric and gastroesophageal junction cancers, did not report on QOL outcomes. ${ }^{11-13}$ Although QOL reported by patients enrolled in randomized clinical trials may not mirror the experiences of patients receiving care in routine clinical settings, it is still vitally important to understand the impact of treatment on QOL, in addition to the common primary endpoint of overall survival. A concerted effort is required to incorporate/mandate QOL endpoints in treatment trials of advanced cancers.

Incorporation of symptom assessment tools in realworld palliative care settings requires systematic implementation through a standardized process by national organizations. In Canada, the accreditation standards for palliative care in oncology are far more granular compared with analogous standards required by the American College of Surgeons Commission on Cancer (CoC). The Canadian accreditation standards for palliative care require the use of standardized tools to assess patient symptoms. ${ }^{14}$ Informative studies such as the study by Merchant et al. are only possible if the symptom assessment tools are widely implemented and the data are appropriately documented in the national database. Although the $\mathrm{CoC}$ has continued to add new standards, it may be time to refine the palliative care requirements to incorporate symptom assessment tools to adjust and improve our treatment approach in advanced cancer patients who are undergoing palliative care.

DISCLOSURES Maheswari Senthil declares no conflict of interest.

\section{REFERENCES}

1. Wagner AD, Syn NL, Moehler M, et al. Chemotherapy for advanced gastric cancer. Cochrane Database Syst Rev. 2017;8:CD004064.
2. Janmaat VT, Steyerberg EW, van der Gaast A, et al. Palliative chemotherapy and targeted therapies for esophageal and gastroesophageal junction cancer. Cochrane Database Syst Rev. 2017;11:CD004063.

3. de Savornin Lohman E, de Bitter T, Verhoeven R, et al. Trends in treatment and survival of gallbladder cancer in the Netherlands; identifying gaps and opportunities from a nation-wide cohort. Cancers (Basel). 2020;12(4):918.

4. Merchant SJ, Kong W, Brundage M, Booth CM. Symptom evolution in patients with esophageal and gastric cancer receiving palliative chemotherapy: a population-based study. Ann Surg Oncol. 2020. https://doi.org/10.1245/s10434-020-09289-6.

5. Kim JW, Kim JG, Kang BW, et al. Treatment patterns and changes in quality of life during first-line palliative chemotherapy in Korean patients with advanced gastric cancer. Cancer Res Treat. 2019;51(1):223-39.

6. Rha SY, Lee J. Symptom clusters during palliative chemotherapy and their influence on functioning and quality of life. Support Care Cancer. 2017;25(5):1519-27.

7. Tsuboi R, Sugishita M, Hirakawa Y, Ando Y. Experiences and hidden needs of older patients, their families and their physicians in palliative chemotherapy decision-making: a qualitative study. Jpn J Clin Oncol. 2020;50(7):779-86.

8. Potgieter T, Maree JE. The palliative chemotherapy decision and experiences of South African patients and their families. Int $J$ Palliat Nurs. 2018;24(6):272-80.

9. Altemus M, Sarvaiya N, Neill Epperson C. Sex differences in anxiety and depression clinical perspectives. Front Neuroendocrinol. 2014;35(3):320-30.

10. American Psychological Association. APA guidelines for psychological practice with boys and men. Washington, DC: American Psychological Association; 2018.

11. Fujitani K, Yang HK, Mizusawa J, et al. Gastrectomy plus chemotherapy versus chemotherapy alone for advanced gastric cancer with a single non-curable factor (REGATTA): a phase 3, randomised controlled trial. Lancet Oncol. 2016;17(3):309-18.

12. Bang YJ, Van Cutsem E, Feyereislova A, et al. Trastuzumab in combination with chemotherapy versus chemotherapy alone for treatment of HER2-positive advanced gastric or gastro-oesophageal junction cancer (ToGA): a phase 3, open-label, randomised controlled trial. Lancet. 2010;376(9742):687-97.

13. Al-Batran SE, Homann N, Pauligk C, et al. Effect of neoadjuvant chemotherapy followed by surgical resection on survival in patients with limited metastatic gastric or gastroesophageal junction cancer: the AIO-FLOT3 trial. JAMA Oncol. 2017;3(9):1237-44.

14. Fassbender K, Watanabe SM. Early palliative care and its translation into oncology practice in Canada: barriers and challenges. Ann Palliat Med. 2015;4(3):135-49.

Publisher's Note Springer Nature remains neutral with regard to jurisdictional claims in published maps and institutional affiliations. 